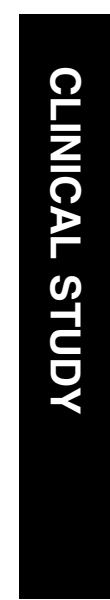

${ }^{1}$ Department of Ophthalmology, Seoul National University College of Medicine, Seoul, Korea

${ }^{2}$ Seoul Artificial Eye Center, Seoul National University Hospital Clinical Research Institute, Seoul, Korea

\section{${ }^{3}$ Department of}

Ophthalmology, Seoul National University Bundang Hospital, Seongnam, Korea

${ }^{4}$ Department of Laboratory Medicine, Seoul National University Bundang Hospital, Seongnam, Korea

Correspondence: KH Park, Department of Ophthalmology, Seoul National University College of Medicine, Seoul National University Bundang Hospital, 300, Gumi-dong, Bundang-gu, Seongnam, Gyunggi-do 463-707, Republic of Korea Tel: + 82317877373 ; Fax: +82 317874057 E-mail: jiani4@ snu.ac.kr

${ }^{5}$ These two authors contributed equally to this work

Received: 26 September 2008

Accepted in revised form: 23 March 2009

Published online: 1 May 2009

Conflict of interest: None

\title{
Serratia marcescens endophthalmitis associated with intravitreal injections of bevacizumab
}

\begin{abstract}
Purpose To report two cases of Serratia marcescens endophthalmitis related to presumed aliquot drug contamination, and to determine the incidence of acute endophthalmitis after intravitreal injection of bevacizumab.
\end{abstract}

Methods A retrospective chart review of 2020 consecutive intravitreal bevacizumab injection (IVBI) cases at the three affiliated hospitals of Seoul National University (A, B, and C) was carried out between 12 October 2006, and 31 January 2008. Bevacizumab was retrieved multiple times from a single original vial as needed and then discarded on the same day at hospital $A$ and $C$, or prepared as a single dose aliquot vial at a compounding pharmacy in the hospital B.

Results The incidence of endophthalmitis after IVBI was $2 / 2020(0.099 \%)$. Two patients receiving IVBI on the same day, but by different surgeons in different sites in hospital $B$, developed acute endophthalmitis. $S$. marcescens was isolated from the vitreous sample of the two patients. Molecular typing with pulsed field gel electrophoresis showed that the organisms were of the same strain, which suggested that the drug was contaminated at the pharmacy. Conclusions Endophthalmitis is a rare complication after IVBI and can be caused by contaminated aliquot drug. Serratia is one of the causative organisms of acute endophthalmitis, which can have devastating consequences, despite the treatment. A compounding pharmacy in a hospital might not be able to guarantee that aliquoted drug is free of contamination for the IVBI.

Eye (2010) 24, 226-232; doi:10.1038/eye.2009.86;

published online 1 May 2009
SH Lee ${ }^{1,2,5}$, SJ Woo ${ }^{1,2,3,5}$, KH Park ${ }^{1,2,3}$, JH Kim, ${ }^{1,2}$, JH Song ${ }^{4}$, KU Park ${ }^{4}$, JW Heo ${ }^{1,2}$, HG Yu ${ }^{1,2}, Y S \mathrm{Yu}^{1,2}$ and $\mathrm{H}$ Chung ${ }^{1,2}$

Keywords: Serratia marcescens; endophthalmitis; bevacizumab; intravitreal injection

Introduction

Intravitreal injections of anti-vascular endothelial growth factor drugs are widely used in the management of ocular diseases, such as exudative age-related macular degeneration, ${ }^{1-3}$ proliferative diabetic retinopathy, ${ }^{4,5}$ diabetic macular oedema, ${ }^{6,7}$ and retinal vein occlusion. ${ }^{8,9}$ One of these drugs, bevacizumab (Avastin; Genentech, South San Francisco, CA, USA), has been frequently used for intraocular injections because of its economic benefits, although it was neither originally developed for ocular diseases nor approved for intraocular use. ${ }^{10}$

Bevacizumab is often divided into aliquots from a large original vial before intravitreal injection to reduce the drug cost per patient. However, this division process always carries the risk of contamination, even in the compounding pharmacy. The reported incidence of endophthalmitis after intravitreal bevacizumab injection (IVBI) ranges from 0.01 to $0.16 \% .{ }^{11-16}$ However, there are no reports regarding endophthalmitis caused by contamination during drug preparation. Furthermore, there are no reports of devastating serratia endophthalmitis causing phthisis after IVBI.

The purpose of this study was to determine the incidence of endophthalmitis after IVBI carried out at three different hospitals, and to describe devastating cases of endophthalmitis caused by Serratia marcescens, presumed to be inoculated during the compounding procedure. 


\section{Materials and methods}

\section{Patients}

This was a retrospective, open-label, uncontrolled, multicenter, consecutive case series. IVBI was carried out in the outpatient office setting or in the operating room in a total of 2020 cases at the three affiliated hospitals (A, B, and C) of Seoul National University, between 12 October 2006 and 31 January 2008. Cases were collected from an injection logbook, and a search for endophthalmitis was conducted among all the cases. The study protocol was approved by the ethics committee of the institution and an informed consent was obtained from all the patients before intravitreal injection.

\section{Injection procedure}

The injection protocol, including pre-injection patient preparation, intravitreal injecting technique, and postinjection management, was standardized in the three hospitals, with the exception of a bevacizumab preparation. After applying topical $0.5 \%$ proparacaine (Alcaine; Alcon, Hünenberg, Switzerland), lid scrubbing and irrigation of the conjunctival sac were carried out with $5 \%$ povidone-iodine. A surgical drape and a sterile lid speculum were applied after periorbital skin scrubbing with $5 \%$ povidone-iodine. In addition, several drops of povidone-iodine were applied and then intravitreal injection was carried out with a 30-gauge needle. Ofloxacin ointment (Tarivid, Santen, Osaka, Japan) was applied after injection. After the procedure, patients were prescribed cefuroxime $250 \mathrm{mg}$, twice a day for 4 days, and levofloxacin $0.5 \%$ (Cravit; Santen, Osaka, Japan), four times a day for 7 days. There were 1078 injections in the hospital A, 600 injections in the hospital $\mathrm{B}$, and 342 injections in the hospital C.

\section{Drug preparations}

The drug division methods were different among the hospitals. In hospital A and C, bevacizumab was aspirated from a single vial of $100 \mathrm{mg}$ per $4 \mathrm{ml}$ into a $1 \mathrm{ml}$ syringe in the injection room of the outpatient clinic, just before intravitreal injection. The surface of the rubber packing of vial was wiped with $10 \%$ povidone-iodine before the insertion of the needle. The same vial was used in the other consecutive injections as needed and was discarded on the same day, after all the injections were complete. In hospital B, one original bevacizumab vial was compounded before injection (0-15 days before injection) into 10 airtight shielded aliquot vials (not the syringe, but the glass bottle with rubber packing) at the compounding pharmacy of the hospital and stored in the refrigerator at $4^{\circ} \mathrm{C}$ before its use. Compounding pharmacy of hospital B has laminar airflow workbenches in the class 7 clean room that is separate from the general pharmacy. Cleaning and sanitizing of the clean room is carried out at regular intervals and routine environmental monitoring was carried out, including routine culture (twice a year). However, maintenance, surveillance, and regular employee training system of the pharmacy did not strictly meet the US pharmacopeia guideline. For example, there was no record that revealed the origin of two aliquot vials.

\section{Identification of the bacterial strain}

To determine whether the bacteria were of the same strain, the isolated serratia were typed with pulsed-field gel electrophoresis (PFGE) (GenePath Strain Typing System, Bio-Rad, Hercules, CA, USA), accompanied by digestion with endonuclease, SpeI. ${ }^{17}$ The PFGE is based on the digestion of DNA extracted from the isolates with restriction enzymes that distinguish few sites in the chromosome. The PFGE patterns were recorded and analysed with a computerized documentation system (Gel Doc Universal Hood 2, Bio-Rad). Isolates, if their PFGE patterns exhibited no fragment differences with each other, were considered as being identical. Isolates were considered to be clonally related if their PFGE patterns exhibited one to three band differences with each other. If the isolates showed a difference of more than six bands, they were considered as being unrelated.

We certify that all applicable institutional and governmental regulations concerning the ethical use of human volunteers were followed during this research.

\section{Results}

There were no cases of endophthalmitis in hospital A and $\mathrm{C}$. Two patients developed acute endophthalmitis in the hospital B. They were injected with bevacizumab aliquots prepared 15 days before the injection. One patient received the injection in the office-based injection room, and the other received it in the elective surgery room. The injections were carried out by two different surgeons. The incidence among the hospitals was not significantly different $(P=0.117$, Table 1$)$. We also compared hospital $A+C$ (using multiple reused original vial) versus hospital B (using compounded aliquot vial) to examine whether any difference depends on the drug preparation methods. This also did not show any significant difference $(P=0.088$, Table 1$)$.

\section{Case reports}

Case 1

A 53-year-old man received IVBI before vitrectomy for diabetic vitreous haemorrhage and tractional retinal 
Table 1 Number of intravitreal bevacizumab injections and incidence of endophthalmitis in the three affiliated hospitals

\begin{tabular}{lcccc}
\hline & Hospital A & Hospital B & Hospital C & Total \\
\hline Number of injections & 1078 & 600 & 342 & 2020 \\
Number of endophthalmitis & 0 & 2 & 0 & 2 \\
Incidence (\%) & 0 & $2 / 600(0.33)$ & 0 & $2 / 2020(0.099)$ \\
\hline
\end{tabular}

Comparison among the tree hospitals $(P=0.117$, Fisher's exact test).

Comparison between hospital A $+\mathrm{C}$ and hospital B $(P=0.088$, Fisher's exact test $)$.

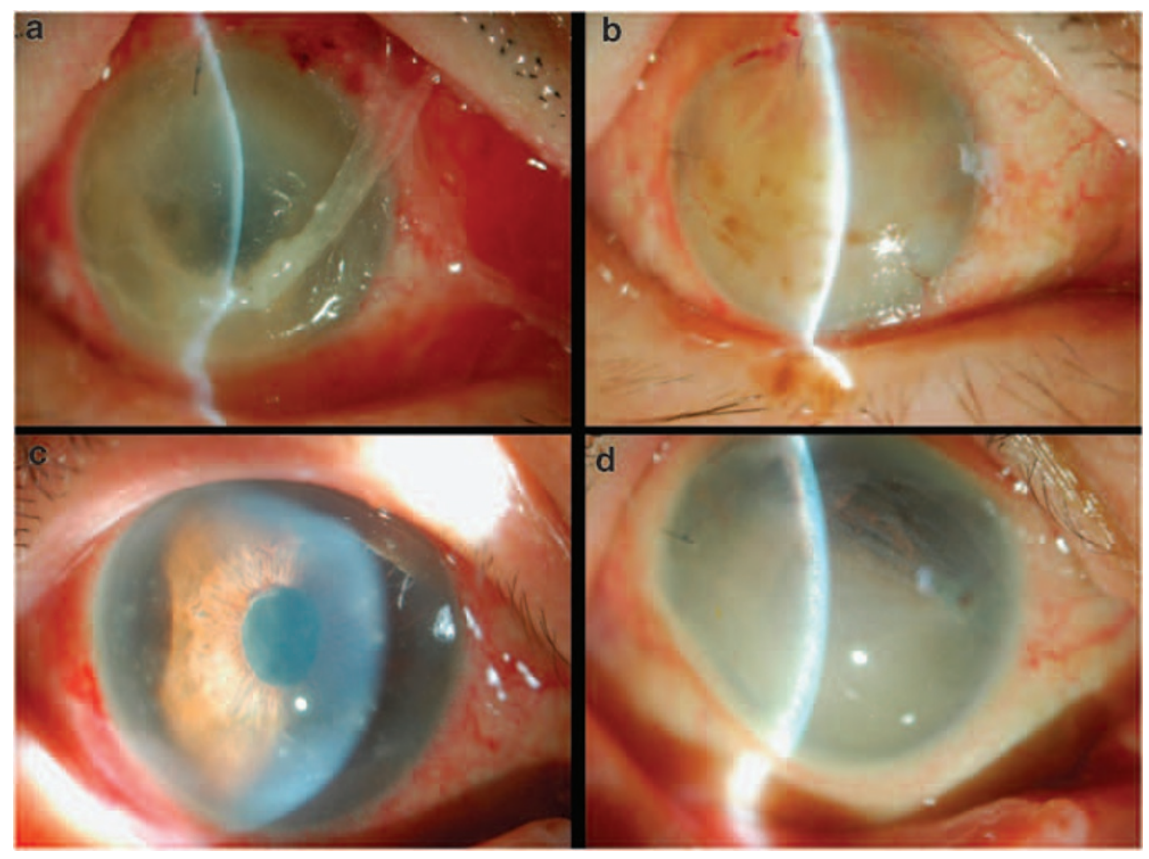

Figure 1 Anterior segment photography of the patients with serratia endophthalmitis. In patient 1, diffuse corneal edema, epithelial defect, and exudation of anterior chamber were observed for 2 days after pars planar vitrectomy (a). The eye went to phthisis at 4 months after PPV (b). In patient 2, fibrinous exudative membrane on lens surface, multiple infiltrates of peripheral cornea, and severe conjunctival infection were observed at 2 days after bevacizumab injection (c). At 4 months after injection, the eye had emulsified silicone oil in the anterior chamber and hypotony. No light perception was observed (d).

detachment in the right eye. His best-corrected visual acuity (BCVA) was 20/200 before the procedure. One day after the IVBI (1.25 mg per $0.05 \mathrm{ml})$, he complained severe ocular pain and the right visual acuity (VA) had decreased to light perception (LP). There were diffuse microcysts on the cornea, $4+$ cells and fibrinous exudative membrane in the anterior chamber. The fundus was invisible because of opaque media. During the preparation time for vitrectomy, intravitreal vancomycin $1.0 \mathrm{mg}$ per $0.1 \mathrm{ml}$ and amikacin $0.4 \mathrm{mg}$ per $0.1 \mathrm{ml}$ injection were administered, and a vitreous biopsy was carried out, all under the presumed diagnosis of infectious endophthalmitis. The patient underwent pars plana vitrectomy (PPV) with vitreous biopsy, lensectomy, fibrinous membrane removal, vancomycin $1.0 \mathrm{mg}$ per $0.1 \mathrm{ml}$ and ceftazidime $2.25 \mathrm{mg}$ per $0.1 \mathrm{ml}$ injection, and silicone oil (SO) injection. During surgery, almost the entire retina was found to be necrotic and covered with a whitish exudative membrane. During the removal of the membrane, the retina was found to be so friable that fullthickness holes were made, even by gentle passive suction with a flute needle. S. marcescens was isolated from the vitreous specimen. Despite four additional intravitreal antibiotic injections and further vitrectomy, the eye had no LP and went on to phthisis 4 months after the IVBI (Figure 1a and b).

\section{Case 2}

A 68-year-old man was treated with IVBI (1.25 mg per $0.05 \mathrm{ml}$ ) in his right eye for exudative age-related macular degeneration on the same day as the patient in case 1. However, IVBI was carried out in the elective operating room by another retinal specialist. The BCVA before the injection was 20/40. At 2 days after the injection, the 
patient complained of decreased VA to counting fingers, along with discomfort in his right eye. He had conjunctival injection, multiple infiltrates in the peripheral cornea, $3+$ cells in the anterior chamber, and a fibrinous membrane on the lens surface (Figure 1c). Vancomycin $1.0 \mathrm{mg}$ per $0.1 \mathrm{ml}$ and ceftazidime $2.25 \mathrm{mg}$ per $0.1 \mathrm{ml}$ were injected into the vitreous cavity, and a vitreous biopsy was carried out. No improvement was noted the following day, and the patient underwent PPV with phacoemulsification, posterior chamber lens insertion, vitreous biopsy, exudative membrane removal, intravitreal vancomycin and ceftazidime injection, and SO injection. Similar to case 1, the entire retina was necrotic and covered with whitish membrane. $S$. marcescens was isolated from the vitreous specimen. Despite repeated intravitreal antibiotic injection, the patient's final VA after 4 months was no LP (Figure 1d).

Both aliquots of bevacizumab used in cases 1 and 2 were derived from two original bevacizumab vials compounded by the hospital pharmacy on the same day. It was not possible to determine whether they were from the same original vial, because all the other 18 aliquots were used within 1 week after the compounding and nothing remained. As the place of injection and surgeon who carried out the injection was different, and the isolated bacteria were identical between the two cases, we suspected that the origin of the infectious agents was not the procedure, but the aliquot drug. The PFGE was carried out to determine whether the bacteria were of the same strain. This test showed that the $S$. marcescens isolated from the two cases were of the same strain (Figure 2).

\section{Discussion}

The incidence of endophthalmitis after IVBI has been found to be up to $0.16 \%$ according to several large case reports. ${ }^{11-16,18}$ The endophthalmitis incidence in our study during a 15-month window was 2/2020 (0.099\%), which was similar to the other reports. It would be hard to compare our series with the others, because of the different injection procedures and the drug preparation methods (Table 2). Furthermore, our endophthalmitis cases might not correlate with the injection procedure, but are related to drug preparation. Despite what we believe to be an exhaustive search of Medline article, we did not find any report addressing the risk of aliquoted bevacizumab contamination and found that this is the first report of Gram-negative bacteria endophthalmitis after IVBI.

The main suspected source of post-injection endophthalmitis has always been ocular surface bacteria $^{21}$ and the most frequent causative organisms of endophthalmitis after IVBI have been conjunctival

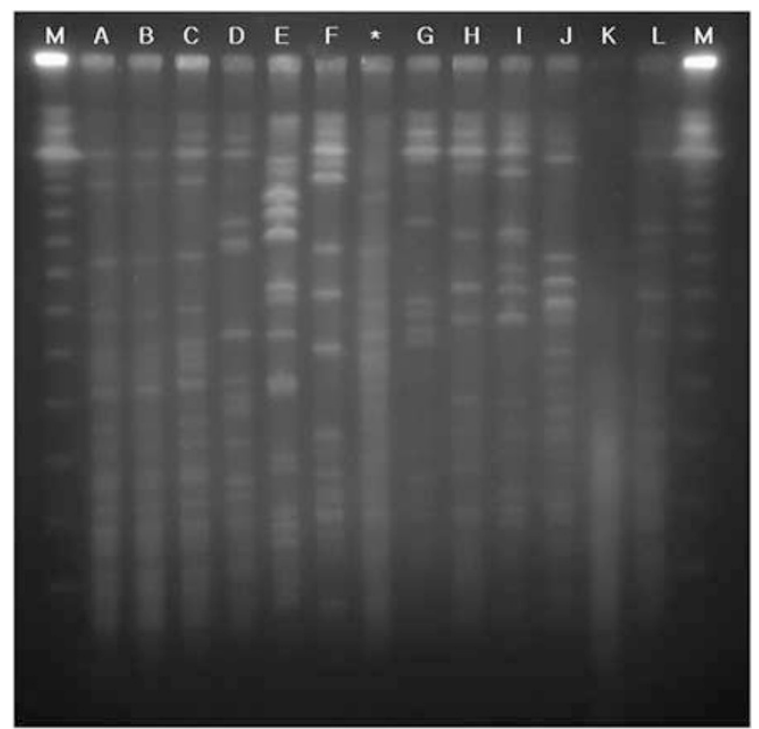

Figure 2 Pulsed-field gel electrophoresis showed that the bacteria isolated from the two patients were of the same strain (A-C). The DNA bands of different strains of Serratia marcescens (D-L) showed different band patterns compared with A, B, and C. (A) Organism cultured and isolated from vitreous biopsy during vitrectomy of case 1. (B) Organism cultured and isolated from vitreous tap of case 2. (C) Organism cultured and isolated from vitreous biopsy during vitrectomy of case 2 . (D-L) S. marcescens of other strains. *Pseudomonas aeruginosa ATCC27853; M. Lambda ladders.

normal flora, including coagulase-negative Staphylococci, Staphylococcus aureus and Streptococcus pneumoniae (Table 2). However, endophthalmitis can be caused by the drug if it is contaminated during the compounding procedure. Earlier reports suggested that bevacizumab injection does not result in an increased risk of injectionrelated complication compared with intravitreal injections with other drugs. ${ }^{12,16,18}$ However, the need to minimize the economic burden on patients necessitates the division of the drug into small aliquots for IVBI, which inevitably introduces a procedural step that may risk contamination and consequent endophthalmitis.

The $S$. marcescens isolated from the two endophthalmitis cases were of the same strain, even though the injections were carried out at different places, at different times, and by different surgeons on the same day. No other surgical procedure-related infection was observed in hospital B. Therefore, we suspected that the drug was contaminated with the organism during the compounding process at the pharmacy. The hospital infection control committee meticulously investigated the compounding procedure at the pharmacy and skin preparation procedure at the clinic and the operation room, but no problem was identifiable with the aseptic techniques, the equipments for compounding, and the instruments for skin preparation procedure. All the 


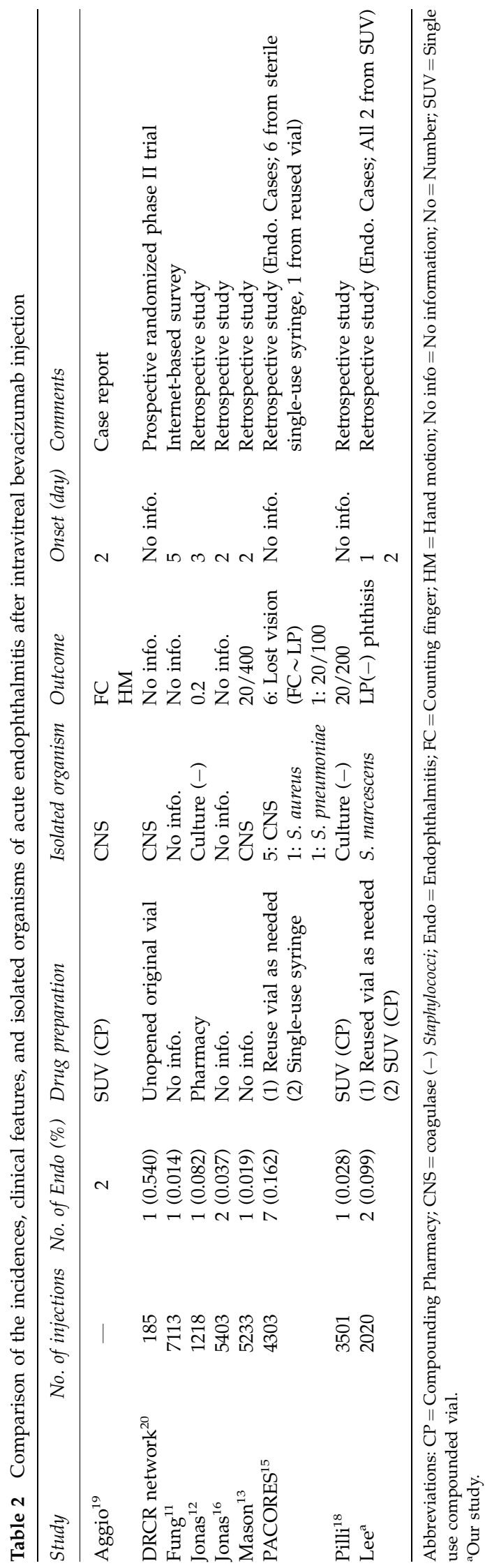

culture results of procedure-related materials were also negative.

It is possible that the other 18 aliquot vials compounded on the same day were also contaminated. All 18 aliquots were injected within 7 days from the compounding procedure, and only these two aliquots that caused endophthalmitis were injected at 15 days after compounding. We hypothesize that the long preservation period (incubation period for bacteria) of the contaminated drug might increase the microbial burden and lead to devastating endophthalmitis. The other possible explanation for the contamination of only two aliquot vials, and not the other 18 aliquot vials, was that the glass bottles used for the two vials were contaminated before or during the compounding procedure. However, it was not possible to prove the hypotheses because no vials were left for further evaluation, and culture results of the compoundingprocedure-related materials were all negative.

Only hospital B that had been using a compounded aliquot vial had cases of endophthalmitis (isolated incidence: $0.33 \%$, Table 1) in this study. Interestingly, PACORES reported a similar result that the rate of the endophthalmits after IVBI was more frequent in the eyes injected using single-use sterile syringes (6/1833 injection; $0.33 \%$ ) than using a single multi-use vial $(0.04 \%)$ that was reused as needed, ${ }^{15}$ even though the compounding method was somewhat different (they used a syringe, but we used a vial). However, there was not enough data to reveal which method was better for the prevention of drug-related endophthalmitis. The best way to reduce drug-related infection seems to be to compound the original bevacizumab vial in a qualified professional compounding pharmacy. However, a professional compounding pharmacy qualified with the US pharmacopeia guideline (USP chapter 797 ) $^{22}$ is often not available in many countries outside the USA, especially for an off-label drug such as bevacizumab. Therefore, many surgeons have no alternative choice to get bevacizumab aliquots except using the compounding pharmacy in their own hospital or using methods, such as drawing multiple doses of bevacizumab out of an original vial, as needed. To prevent the infection originated from the inappropriate compounding, routine contamination surveillance, such as random microbiological assay, sample retention, and annual certification of staff, is mandatory as a qualified compounding pharmacy does.

The S. marcescens endophthalmitis has been reported as a result of endogenous metastatic infection and after intraocular surgery. ${ }^{23,24}$ The sequel of $S$. marcescens endophthalmitis are usually devastating. The patients in our cases lost their vision and went to phthisis despite the surgical treatment. Even though the incidence of 
endophthalmitis was rare and not significantly different among the hospitals, caution must be exercised to prevent it, because the endophthalmitis can be devastating depending on the virulence of the infected bacteria. ${ }^{19,25,26}$

This study is limited by several factors, including retrospective design, short-term period, different drug preparation methods, lack of comparison with other drugs, and no randomization among the surgeons, but is providing some information of warning that inappropriate compounding can cause a devastating endophthalmitis depending on the virulence of the infected organism. Another concern that must be considered is that phacoemulsification with intraocular lens (IOL) insertion was carried out in case 2. IOL should not be placed in patients with highly suspicious active infection. We tried to remove IOL at the end of the surgery, but IOL removal was not possible because of iris bleeding. This might worsen the prognosis of endophthalmitis in case 2.

After these two cases, we converted the drug division method of hospital $B$ to be similar to that of the other two hospitals $(\mathrm{A}, \mathrm{C})$ : multiple retrieval of an original vial just before the procedure and disposal on the same day. We routinely carried out microbiological testing with the remnant bevacizumab vials before discarding and have not found any positive results till now.

In conclusion, acute bacterial endophthalmitis is a rare complication after IVBI with an incidence of about 1 case per 1000 injections. Serratia, which happened to be the causative organism in our cases, led to devastating outcomes. Our experience suggests that the contamination may occur even in compounding pharmacies. In addition to proper preparations of intravitreal injection procedure, surgeons should be cautious to use a compounded drug and should consider a routine contamination surveillance system for drug compounding procedure, if a qualified professional compounding pharmacy is not available.

\section{References}

1 Csaky K. Anti-vascular endothelial factor for neovascular age related macular degeneration. Ophthalmology 2003; 110: 879-881.

2 Gragoudas ES, Adamis AP, Cunningham Jr ET, Feinsod M, Guyer DR, VISION Clinical Trial Group. Pegaptanib for neovascular age-related macular degeneration. $N$ Engl J Med 2004; 351(27): 2805-2816.

3 Rosenfeld PJ, Brown DM, Heier JS, Boyer DS, Kaiser PK, Chung CY et al. Ranibizumab for neovascular age-related macular degeneration. $N$ Engl J Med 2006; 355(14): 1419-1431.

4 Avery RL, Pearlman J, Pieramici DJ, Rabena MD, Castellarin AA, Nasir MA et al. Intravitreal bevacizumab (Avastin) in the treatment of proliferative diabetic retinopathy. Ophthalmology 2006; 113(10): 1695-1705.

5 Jorge R, Costa RA, Calucci D, Cintra LP, Scott IU. Intravitreal bevacizumab (Avastin) for persistent new vessels in diabetic retinopathy (IBEPE study). Retina 2006; 26(9): 1006-1013.

6 Cunningham Jr ET, Adamis AP, Altaweel M, Aiello LP, Bressler NM, D'Amico DJ et al. A phase II randomized double-masked trial of pegaptanib, an anti-vascular endothelial growth factor aptamer, for diabetic macular edema. Ophthalmology 2005; 112(10): 1747-1757.

7 Haritoglou C, Kook D, Neubauer A, Wolf A, Priglinger S, Strauss $\mathrm{R}$ et al. Intravitreal bevacizumab (Avastin) therapy for persistent diffuse diabetic macular edema. Retina 2006; 26(9): 999-1005.

8 Jaissle GB, Ziemssen F, Petermeier K, Szurman P, Ladewig $\mathrm{M}$, Gelisken $\mathrm{F}$ et al. Bevacizumab for treatment of macular edema secondary to retinal vein occlusion. Ophthalmologe 2006; 103(6): 471-475.

9 Wu L, Arevalo JF, Roca JA, Maia M, Berrocal MH, Rodriguez FJ et al. Comparison of two doses of intravitreal bevacizumab (Avastin) for treatment of macular edema secondary to branch retinal vein occlusion: results from the Pan-American Collaborative Retina Study Group at 6 months of follow-up. Retina 2008; 28(2): 212-219.

10 Chen HX, Gore-Langton RE, Cheson BD. Clinical trials referral resource: current clinical trials of the anti-VEGF monoclonal antibody bevacizumab. Oncology (Williston Park) 2001; 15(8): 1017-1026.

11 Fung AE, Rosenfeld PJ, Reichel E. The International Intravitreal Bevacizumab Safety Survey: using the internet to assess drug safety worldwide. $\mathrm{Br} J$ Ophthalmol 2006; 90(11): 1344-1349.

12 Jonas JB, Spandau UH, Rensch F, Von Baltz S, Schlichtenbrede $\mathrm{F}$ et al. Infectious and noninfectious endophthalmitis after intravitreal bevacizumab. J Ocul Pharmacol Ther 2007; 23(3): 240-242.

13 Mason III JO, White MF, Feist RM, Thomley ML, Albert MA, Persaud TO et al. Incidence of acute onset endophthalmitis following intravitreal bevacizumab (Avastin) injection. Retina 2008; 28(4): 564-567.

14 Meyer $\mathrm{CH}$, Mennel S, Eter N. Incidence of endophthalmitis after intravitreal Avastin injection with and without postoperative topical antibiotic application. Ophthalmologe 2007; 104(11): 952-957.

15 Wu L, Martinez-Castellanos MA, Quiroz-Mercado H, Arevalo JF, Berrocal MH, Farah ME et al. Twelve-month safety of intravitreal injections of bevacizumab (Avastin(R)): results of the Pan-American Collaborative Retina Study Group (PACORES). Graefes Arch Clin Exp Ophthalmol 2008; 246(1): 81-87.

16 Jonas JB, Spandau Uh, Schlichtenbrede F. Short-term complications of intravitreal injections of triamcinolone and bevacizumab. Eye 2008; 22: 590-591.

17 Tenover FC, Arbeit RD, Goering RV, Mickelsen PA, Murray $\mathrm{BE}$, Persing DH et al. Interpreting chromosomal DNA restriction patterns produced by pulsed-field gel electrophoresis: criteria for bacterial strain typing. J Clin Microbiol 1995; 33: 2233-2239.

18 Pilli S, Kotsolis A, Spaide RF, Slakter J, Freund KB, Sorenson $\mathrm{J}$ et al. Endophthalmitis associated with intravitreal antivascular endothelial growth factor therapy injections in an office setting. Am J Ophthalmol 2008; 145: 879-882. 
19 Aggio FB, Farah ME, de Melo GB, d'Azevedo PA, Pignatari AC, Höfling-Lima AL. Acute endophthalmitis following intravitreal bevacizumab (Avastin) injection. Eye 2007; 21(3): 408-409.

20 Diabetic Retinopathy Clinical Research Network. A phase II randomized clinical trial of intravitreal bevacizumab for diabetic macular edema. Ophthalmology 2007; 114: 1860-1867.

21 Speaker MG, Milch FA, Shah MK, Eisner W, Kreiswirth BN Role of external bacterial flora in the pathogenesis of acute postoperative endophthalmitis. Ophthalmology 1991; 98(5): 639-649.

22 Kastango ES, American Society of Health-System Pharmacists (ASHP). Blueprint for implementing USP Chapter 797 for compounding sterile preparations. Am J Health Syst Pharm 2005; 62: 1271-1288.
23 Kumar N, Gandhewar R, Khan MY. Serratia marcescens endophthalmitis after cataract surgery despite vancomycin and gentamicin in irrigation fluid. Can J Ophthalmol 2004; 39(7): 778-779.

24 Radda TM. Metastatic Serratia marcescens endophthalmitis. Ophthalmologica 1982; 185(2): 65-68.

25 Alkuraya HS, Al-Kharashi AS, Alharthi E, Chaudhry IA. Acute endophthalmitis caused by Staphylococcus lugdunesis after intravitreal bevacizumab

(Avastin) injection. Int Ophthalmol 2008; E-pub ahead of print.

26 Kopel AC, Carvounis PE, Holz ER. Bacillus cereus endophthalmitis following intravitreous bevacizumab injection. Ophthalmic Surg Lasers Imaging 2008; 39(2): 153-154. 